\title{
Cardiopulmonary bypass and halogenated agents
}

\author{
Guillermo Lema ${ }^{1} \mathbb{B}$
}

Received: 23 November 2020 / Accepted: 18 December 2020 / Published online: 7 January 2021

(c) Japanese Society of Anesthesiologists 2021

To the Editor:

I have read with interest the manuscript by Takahiro Tamura et al [1].

I would like to add some comments to the discussion.

It is interesting to know what happens with blood levels with the use of those agents during cardiac surgery, it adds important information.

a. This is considered by the authors a "pilot study", so the number of patients undergoing the study is small to infer proper and robust conclusion.

b. It would have been important to have samples at different times during CPB. We do not know as yet if blood levels of the halogenated agents may vary during hypothermia, rewarming or over time. Isoflurane behaves the same way?

c. There is no reason to believe that the agents could produce any damage to these oxygenators. They have been used for many years, with success, and the damage they may present is due to multifactorial causes, and reflects most of the time anomalous situations during CPB.

d. With the new monitoring devices, we can be aware of the hypnotic effects of many drugs on patients. So now, we can control closely the agents we are using. Studies such as these are important. As suggested, samples are needed at different times and studies should include a larger sample size.

\section{Reference}

1. Tamura T, Mori A, Ishii A, Ando M, Nishiwaki K. Desflurane and sevoflurane concentrations in blood passing through the oxygenator during cardiopulmonary bypass: a randomized prospective pilot study. J Anesth. 2020;34:904-11.

Publisher's Note Springer Nature remains neutral with regard to jurisdictional claims in published maps and institutional affiliations.

This comment refers to the article available online at https://doi. org/10.1007/s00540-020-02844-1.

Guillermo Lema

glema@med.puc.cl

1 Anesthesiology Division, Pharmacology and Toxicology Department, Facultad de Medicina, Pontificia Universidad Catolica de Chile, Marcoleta 377, Santiago, Chile 